\title{
The combinatorics of knot invariants arising from the study of Macdonald polynomials
}

\author{
J. Haglund Department of Mathematics \\ University of Pennsylvania, Philadelphia, PA 19104-6395 \\ jhaglund@math. upenn.edu
}

June 25, 2015

\begin{abstract}
This chapter gives an expository account of some unexpected connections which have arisen over the last few years between Macdonald polynomials, invariants of torus knots, and lattice path combinatorics. The study of polynomial knot invariants is a well-known branch of topology which originated in the 1920's with the one-parameter Alexander polynomial [Ale28]. In the early 1980's Jones [Jon85] introduced a different one-parameter polynomial invariant, with important connections to physics. Shortly thereafter a number of authors more or less simultaneously discovered the HOMFLY polynomial, a two-parameter invariant which includes both the Alexander and Jones polynomials as special cases. The HOMFLY polynomial can be calculated recursively through skein relations. In the late 1980's Witten showed that the Jones polynomial and related invariants have an interpretation in terms of Chern-Simons theory, which is central to string theory.

In 2006 Dunfield, Gukov, and Rasmussen [DGR06] hypothesized the existence of a threeparameter knot invariant, now known as the "superpolynomial knot invariant" of a knot $K$, denoted $\mathcal{P}_{K}(a, q, t)$, which includes the HOMFLY polynomial as a special case. Since then various authors proposed different possible definitions of the superpolynomial, which are conjecturally all equivalent. These definitions typically involve homology though, and are difficult to compute.

In the case of torus knots an accepted definition of the superpolynomial has recently emerged from work of Aganagic and Shakirov [AS12], [AS11] (using refined Chern-Simons theory) and Cherednik [Che13] (using the double affine Hecke algebra). Gorsky and Negut [GN13] showed that these two different constructions yield the same three parameter knot invariant which is now accepted as the definition of the superpolynomial for torus knots. These constructions involve symmetric functions in a set of variables $X$ known as Macdonald polynomials, which also depend on two extra parameters $q, t$. These symmetric functions are important in algebraic combinatorics and other areas, and play a central role in various character formulas for $S_{n}$ modules connected to the Hilbert scheme from algebraic geometry. In particular, Haiman's formula for the bigraded character of the space $\mathrm{DH}_{n}$ of diagonal harmonics under the diagonal action of the symmetric group is expressed in terms of Macdonald polynomials.

E. Gorsky [Gor12], [ORS12, Appendix] noticed that the coefficient of $a^{j}$ in the superpolynomial of the $(n+1, n)$ torus knot equals the bigraded multiplicity of a certain hook shape in the character of $\mathrm{DH}_{n}$. This polynomial is known as the $(q, t)$-Schröder polynomial since the author showed it can be expressed as a weighted sum over Schröder lattice paths in the $n \times n+1$ rectangle. Gorsky and Negut have shown that the coefficient of $a^{j}$ in the superpolynomial of the $(m, n)$ torus knot can be viewed as the coefficient of a certain hook Schur function in a symmetric function expression involving Macdonald polynomials, and they
\end{abstract}


have derived many explicit identities for this object. In addition Oblomkov, Rasmussen, and Shende [ORS12] have introduced a conjectured extension of the $q, t$-Schröder polynomial to general $(m, n)$ giving a positive strictly combinatorial expression for the superpolynomial of the $(m, n)$ torus knot. This conjecture connects nicely with an important conjecture in algebraic combinatorics called the rational shuffle conjecture. In the following pages we will describe these developments in more detail.

\section{Knot Invariants}

A knot is an embedding of a circle in $\mathbf{R}^{3}$. Informally, we will think of a knot as a closed, elastic string in $\mathbf{R}^{3}$. Two knots are equivalent (isotopic) if one string can be deformed into the other without cutting the string. Our knots will carry an orientation, i.e. a forward direction indicated by an arrow. Knots are typically represented by planar diagrams called knot diagrams, as in Figure 1.

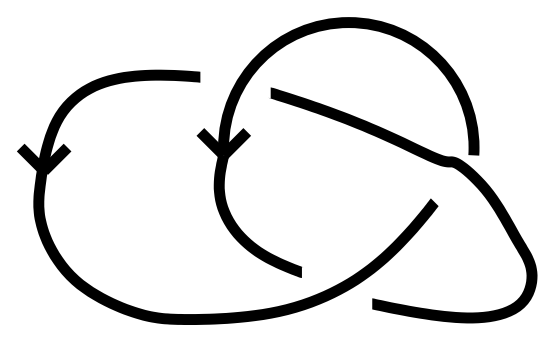

Left handed trefoil

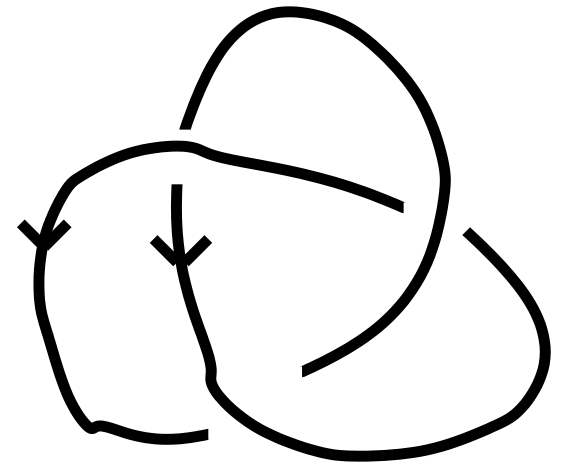

Right handed trefoil

Figure 1: Knot diagrams of the two trefoil knots

The trefoil knot is the simplest example of a knot which is not isotopic to the unknot $O$ (a closed string with no crossings). The right handed trefoil is the mirror image of the left handed trefoil. It is known that the trefoil knot is not isotopic to its mirror image, i.e. is not chiral. An interesting family of knots that will be important to us are torus knots. Given two relatively prime positive integers $(m, n)$, we let $T_{(m, n)}$ denote the corresponding torus knot, which can be obtained by wrapping a string around a torus, at such an angle that by the time you return to where you started from you have wrapped around the torus $n$ times in one direction and $m$ times in the other. It is known that $T_{(m, n)}$ is isotopic to $T_{(n, m)}$, and that $T_{(3,2)}$ is the left handed trefoil.

A (polynomial) knot invariant is a polynomial in (possibly) several variables which is the same for isotopic knots. In the 1920's Alexander [Ale28] introduced the first polynomial knot invariant, which he constructed from a knot diagram by taking the determinant of a certain $n \times n$ matrix, where $n$ is the number of crossings in the diagram of the knot. In 1969 Conway [Con70] showed that a version of this polynomial could be computed recursively using skein relations. 
Specifically, the Alexander-Conway polynomial $\mathrm{AC}_{L}(z)$ of the knot $L$ satisfies

$$
\mathrm{AC}_{L_{+}}(z)=\mathrm{AC}_{L_{-}}(z)+z \mathrm{AC}_{L_{0}}(z)
$$

where $L_{+}, L_{-}$, and $L_{0}$ indicate knots which are the same everywhere except at a specific crossing, see Figure 2. We use the initial condition $\operatorname{AC}_{O}(z)=1$, where $O$ is the "unknot" consisting of a circle with no crossings. We note that our recursive definition actually applies to oriented links, which can be represented by knot diagrams consisting of possibly more than one string.
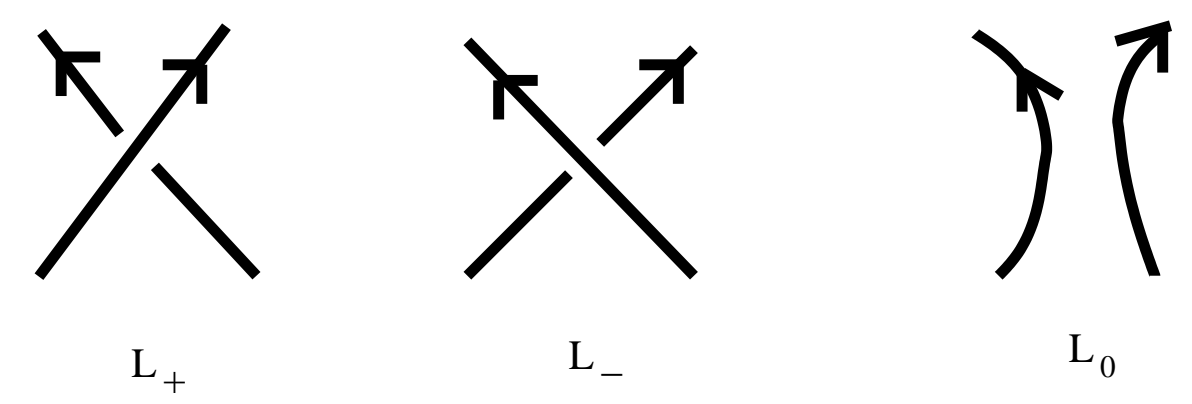

Figure 2: The three types of crossings in a knot diagram

Although the same knot can be represented by a knot diagram in many different ways, the polynomial $\mathrm{AC}_{L}(z)$ turns out to be independent of the diagram.

Example 1 Let $T$ denote the left handed trefoil knot of Figure 1. In this example we calculate $A C_{T}(z)$ using the skein relations. (We follow the procedure carried out in [Wik] for the right handed trefoil). Now

$$
A C_{T}(z)=A C_{O}(z)+z A C_{H}(z)
$$

where $H$ is the Hopf link as in Figure 3.

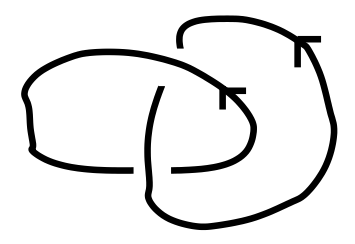

Figure 3: One of the two oriented Hopf links, which occurs in the computation of $\mathrm{AC}_{T}(z)$.

Furthermore, we have

$$
A C_{H}=A C_{O_{2}}+z A C_{O}
$$

where $\mathrm{O}_{2}$ is the union of two unknots (called the unlink of two components). To find $A C_{\mathrm{O}_{2}}(z)$ we use the identity in Figure 4, which shows $A C_{O_{2}}(z)=0$, since the two other knots are both the unknot $O$. Putting everything together we have

$$
A C_{T}(z)=1+z(0+z)=1+z^{2} .
$$



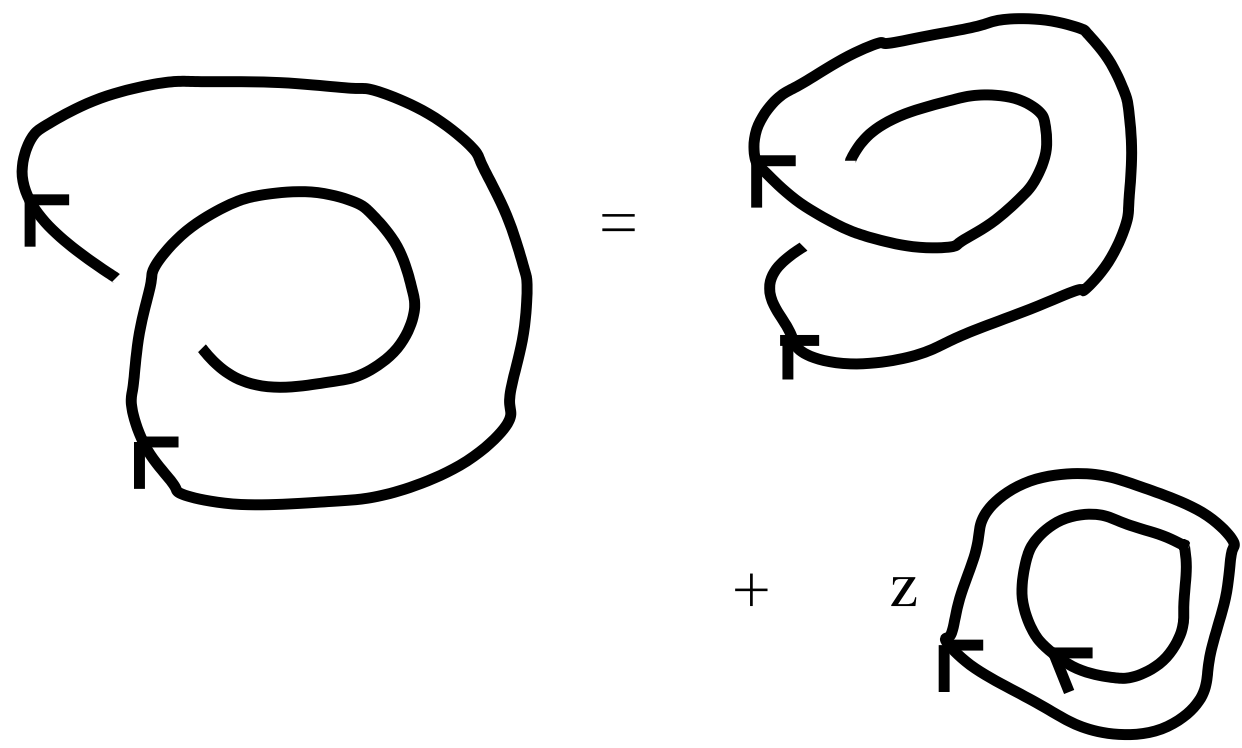

Figure 4: A recursive relation involving the unlink $O_{2}$

Jones [Jon85] introduced a different knot invariant $V_{L}(z)$, which can also be defined recursively via the relation

$$
z V_{L_{+}}(z)=\frac{1}{z} V_{L_{-}}(z)+\left(\frac{1}{\sqrt{z}}-\sqrt{z}\right) V_{L_{0}}(z)
$$

together with the initial condition $V_{O}(z)=1$. Shortly after this three different pairs of authors simultaneously discovered the HOMFLY polynomial $P_{K}(\alpha, z)$, a two-variable knot invariant which contains the Jones and Alexander-Conway polynomials as special cases. These three pairs of authors each submitted papers on this to the Bulletin of the AMS, and the editor convinced them to combine their articles into one $\left[\mathrm{FYH}^{+} 85\right]$. (The word HOMFLY is a combination of the first letters of the last names of the six authors.) Their polynomial can be defined recursively as above by

$$
\alpha P_{L_{+}}(\alpha, z)=\frac{1}{\alpha} P_{L_{-}}(\alpha, z)+z P_{L_{0}}(\alpha, z) .
$$

If we compute $P_{T_{(3,2)}}(\alpha, z)$ as in Example 1, using (6), we get

$$
P_{T_{(3,2)}}(\alpha, z)=P_{0}(\alpha, z)\left(2 \alpha^{2}+\alpha^{2} z^{2}-\alpha^{4}\right) .
$$

Different initial values for the HOMFLY polynomial $P_{0}(\alpha, z)$ are used by various authors.

In 2006 Dunfield, Gukov, and Rasmussen [DGR06] hypothesized the existence of a threeparameter knot invariant, the so-called superpolynomial of a knot $K$, denoted $\mathcal{P}_{K}(a, q, t)$, which includes the HOMFLY polynomial as a special case. They were not able to define the superpolynomial in general, but listed various desirable properties which it should satisfy, and showed that for many small knots there was a unique polynomial with these properties. Over the next several years various authors proposed different possible definitions of the superpolynomial, which 
are conjecturally all equivalent. These definitions typically involve homology though, and are difficult to compute.

In 2011 Aganagic and Shakirov [AS12], [AS11] used refined Chern-Simons theory to construct knot invariants of torus knots. Cherednik [Che13] gave an alternate way to construct knot invariants for these knots using the double affine Hecke algebra. Gorsky and Negut [GN13] showed that these two different constructions gave the same knot invariants. A certain stable limit of these invariants yields a three parameter knot invariant which is now accepted as the definition of the superpolynomial for torus knots.

E. Gorsky [Gor12] made the exciting discovery that the superpolynomial of the $(n+1, n)$ torus knot, has an interpretation in terms of the character of $\mathrm{DH}_{n}$. To be precise, if we let $\tilde{P}_{T_{(m, n)}}(a, q, 1 / t)$ be the modified superpolynomial (obtained from the superpolynomial by replacing $t$ by $1 / t)$ then as he showed [ORS12, Appendix] that the coefficient of $a^{k}$ in $\tilde{\mathcal{P}}_{T_{(n+1, n)}}(a, q, 1 / t)$ equals the scalar product

$$
(-1)^{k}\left\langle\nabla e_{n}, e_{n-k} h_{k}\right\rangle
$$

Here $\nabla e_{n}$ is Haiman's formula for the character of $\mathrm{DH}_{n}$, described in more detail in Section 3, $e_{k}$ is the $k$ th elementary symmetric function, $h_{k}$ is the $k$ th homogeneous symmetric function, and $\langle$,$\rangle is the Hall scalar product, with respect to which the Schur functions are orthonormal.$ There is a known combinatorial formula for (8) due to the author [Hag04], which is expressed in terms of weighted lattice paths.

Inspired by the study of the Hilbert scheme of the plane curve $x^{n}=y^{m}$, Oblomkov, Rasmussen, and Shende [ORS12] have introduced a conjectured expression for a certain Poincaré polynomial associated to knot homology. Their formula gives a candidate positive combinatorial formula for the superpolynomial of the $(m, n)$ torus knot, which reduces to (8) when $m=n+1$.

In a parallel development, a natural generalization of the symmetric function $\nabla e_{n}$ (the character of $\mathrm{DH}_{n}$ ) has recently emerged from work of Burban, Schiffman, Vasserot, Negut and others [BS12], [SV11], [SV13], [Neg14] on the Elliptic Hall algebra and other related objects in algebraic geometry and string theory. These symmetric functions (denoted here by $\left.Q_{(m, n)}(-\mathbf{1})^{\mathbf{n}}\right)$ depend on a pair of positive integers $(m, n)$, and can be defined recursively (see [BGLX14b] for an explicit description of the symmetric function operators $\left.Q_{(m, n)}\right)$. There is a nice conjecture for their expansion into monomials [GN13], [BGLX14a] called the rational shuffle conjecture, which reduces to the shuffle conjecture in $\left[\mathrm{HHL}^{+} 05 \mathrm{c}\right]$ when $m=n+1$. Gorsky and Negut [GN13] have shown that

$$
\tilde{P}_{T_{(m, n)}}(a, q, t)=(-1)^{k}\left\langle Q_{(m, n)}(-\mathbf{1})^{\mathbf{n}}, e_{n-k} h_{k}\right\rangle .
$$

The conjectured combinatorial formula for $\tilde{\mathcal{P}}_{T_{(m, n)}}(a, q, t)$ in [ORS12] can be viewed as the "hook case" of the rational shuffle conjecture, since the scalar product of $e_{n-k} h_{k}$ with a symmetric function $F$ is the sum of the coefficients of the two consecutive hook shapes $s_{k, 1^{n-k}}$ and $s_{k+1,1^{n-k-1}}$ in $F$. (Eq. (9) implies a corresponding formula for the coefficient of a single hook shape in $\left.Q_{n, m}(-\mathbf{1})^{\mathbf{n}}\right)$.

All of these constructions involve advanced symmetric functions with two extra parameters $q, t$ known as Macdonald polynomials, which are the subject of the next section. 


\section{Macdonald polynomials}

Let $p_{k}(X)=\sum_{i} x_{i}^{k}$ be the $k$ th power sum, and for any polynomial $E\left(t_{1}, t_{2}, \ldots, t_{n}\right)$ define the plethystic substitution of $E$ into $p_{k}$ as

$$
p_{k}[E]=E\left(t_{1}^{k}, \ldots, t_{n}^{k}\right) .
$$

For example,

$$
\begin{array}{r}
p_{k}[X(1-t)]=\sum_{i} x_{i}^{k}\left(1-t^{k}\right)=p_{k}(X)\left(1-t^{k}\right) \\
p_{k}[X /(1-t)]=p_{k}(X) /\left(1-t^{k}\right) .
\end{array}
$$

So when plethystically substituting $E$ into $p_{k}$, we replace any variables by their $k$ th powers, and leave any constants like -1 alone. To plethystically substitute $E$ into an arbitrary symmetric function $f$, we first express $f$ as a polynomial in the $p_{k}$, then substitute $E$ into each $p_{k}$ in this expression. See [Hag08, pp.19-20] for more background on plethystic substitution.

In 1988 Macdonald [Mac88], [Mac95] introduced symmetric functions $P_{\mu}(X ; q, t)$ which satisfy an orthogonality condition, and include many previously studied bases of symmetric functions as special cases. For example, $P_{\mu}(X ; q, q)=s_{\mu}(X)$ (for any $q$ ), and $P_{\mu}(X ; 0, t)$ is the Hall-Littlewood polynomial. Macdonald also defined the integral form $J_{\mu}$ as

$$
J_{\mu}(X ; q, t)=\prod_{x \in \mu}\left(1-q^{a} t^{l+1}\right) P_{\mu}(X ; q, t)
$$

with $a, l, a^{\prime}, l^{\prime}$ denoting the arm, leg, coarm, coleg of the cell, as in Figure 5.

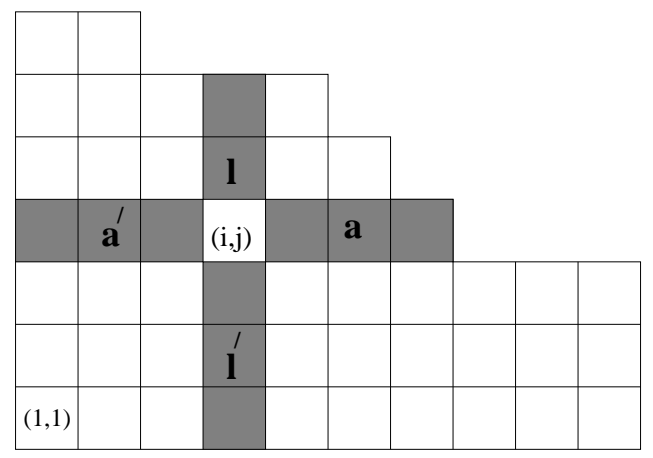

Figure 5: The $\operatorname{arm} a$, coarm $a^{\prime}, \operatorname{leg} l$ and coleg $l^{\prime}$ of a cell

Macdonald conjectured that when $J_{\mu}$ is expanded in terms of the "plethystic Schur basis" $\left\{s_{\lambda}[X(1-t)]\right\}$ the coefficients are all positive, i.e are in $\mathbb{N}[q, t]$. Garsia and Haiman [GH93] introduced the modified Macdonald polynomials $\tilde{H}_{\mu}(X ; q, t)$;

$$
\tilde{H}_{\mu}[X ; q, t]=t^{n(\mu)} J_{\mu}\left[\frac{X}{1-1 / t} ; q, 1 / t\right]
$$

and gave a conjectured interpretation for $\tilde{H}_{\mu}(X ; q, t)$ as the bigraded character of a certain $S_{n}$ module $V_{\mu}$. This refined Macdonald's positivity conjecture since it implies the coefficient of $s_{\lambda}$ 
in $\tilde{H}_{\mu}$ counts multiplicities of irreducible representations and is hence positive, and this implies a similar interpretation for the coefficient of $s_{\lambda}[X(1-t)]$ in $J_{\mu}$. The conjecture of Garsia and Haiman became known as the $n$ ! conjecture since it reduced to the problem of showing that the dimension of $V_{\mu}$ is $n$ !, for $\mu$ any partition of $n$.

After ten years of intensive research, Haiman [Hai01] proved the $n$ ! conjecture using the Hilbert scheme from algebraic geometry. Finding a positive, combinatorial formula for the Schur coefficients in $\tilde{H}_{\mu}$ is still an important open problem. Haiman's interpretation of $\tilde{H}_{\mu}$ in terms of the Hilbert scheme has had a growing influence on algebraic geometry and string theory, particularly in the construction of character formulas expressed in terms of Macdonald polynomials.

\section{Diagonal Harmonics and Rational Catalan Combinatorics}

The space of diagonal harmonics $D H_{n}$ is defined as

$$
D H_{n}=\left\{f \in \mathbb{C}\left[X_{n}, Y_{n}\right]: \sum_{i=1}^{n} \frac{\partial^{h}}{x_{i}^{h}} \frac{\partial^{k}}{y_{i}^{k}} f=0, \forall h+k>0 .\right\} .
$$

The symmetric group $S_{n}$ acts "diagonally" on $\mathrm{DH}_{n}$ by permuting the $X$ and $Y$ variables in the same way;

$$
\sigma f\left(x_{1}, \ldots x_{n}, y_{1}, \ldots, y_{n}\right)=f\left(x_{\sigma_{1}}, \ldots, x_{\sigma_{n}}, y_{\sigma_{1}}, \ldots, y_{\sigma_{n}}\right) \quad \forall \sigma \in S_{n} .
$$

Example 2 An explicit basis for $D H_{2}$ is given by $\left\{1, x_{2}-x_{1}, y_{2}-y_{1}\right\}$. Taking into account the $S_{n}$-action, we see that $x_{2}-x_{1}$ forms a sign-character component, as does $y_{2}-y_{1}$, and 1 forms a trivial character component. If we weight our components by $q^{x \text {-degree }} t^{y \text {-degree }}$, then the Hilbert series of $\mathrm{DH}_{2}$ is $1+q+t$, and the Frobenius series (where as usual the Schur function $s_{\lambda}$ is used to mark the irreducible component corresponding to $\lambda)$ is $s_{2}+(q+t) s_{1^{2}}$.

Garsia and Haiman initiated the study of $\mathrm{DH}_{n}$ [Hai94], [GH96], and conjectured that the Frobenius series $\mathrm{DH}_{n}$ can be expressed compactly as $\nabla e_{n}(X)$. Here $\nabla$ is a linear operator on symmetric functions defined on the $\tilde{H}_{\mu}$ basis via

$$
\nabla \tilde{H}_{\mu}=t^{n(\mu)} q^{n\left(\mu^{\prime}\right)} \tilde{H}_{\mu}
$$

where $n(\mu)=\sum_{i}(i-1) \mu_{i}$. Haiman [Hai02] proved this conjecture. His proof uses the Hilbert scheme and other elements of his proof of the $n$ ! Theorem.

It follows from Macdonald's original work that

$$
\nabla e_{n}=\sum_{\mu \vdash n} \frac{t^{n(\mu)} q^{n\left(\mu^{\prime}\right)} M \tilde{H}_{\mu} \Pi_{\mu} B_{\mu}}{w_{\mu}},
$$

where $B_{\mu}=\sum_{s \in \mu} q^{a^{\prime}} t^{l^{\prime}}, M=(1-q)(1-t), \Pi_{\mu}=\prod_{s \in \mu, s \neq(1,1)}\left(1-q^{a^{\prime}} t^{l^{\prime}}\right)$, and $w_{\mu}=\prod_{s \in \mu}\left(q^{a}-\right.$ $\left.t^{l+1}\right)\left(t^{l}-q^{a+1}\right)$. Although everything in (18) is explicit, the formula is difficult to interpret combinatorially.

Let $C_{n}(q, t)=\left\langle\nabla e_{n}, s_{1^{n}}\right\rangle$, the sign character of $\mathrm{DH}_{n}$. This polynomial is particularly interesting combinatorially, since $C_{n}(1,1)=C_{n}$, the $n$th Catalan number. We now give a purely combinatorial description of $C_{n}(q, t)$. 
Definition 1 Let $L^{+}(m, n)$ denote the set of lattice paths in the first quadrant of the xy-plane, consisting of unit North $N$ and East $E$ steps, which start at $(0,0)$, end at $(m, n)$, and never go below the main diagonal $n x=m y$. Given $\pi \in L_{n, n}^{+}$, define the bounce path of $\pi$ to be the path described by the following algorithm.

Start at $(0,0)$ and travel North along $\pi$ until you encounter the beginning of an E step. Then turn East and travel straight until you hit the diagonal $y=x$. Then turn North and travel straight until you again encounter the beginning of an $E$ step of $\pi$, then turn East and travel to the diagonal, etc. Continue in this way until you arrive at $(n, n)$.

We can think of our bounce path as describing the trail of a billiard ball shot North from $(0,0)$, which "bounces" right whenever it encounters a horizontal step and "bounces" up when it encounters the line $y=x$. The bouncing ball will strike the diagonal at places

$$
(0,0),\left(j_{1}, j_{1}\right),\left(j_{2}, j_{2}\right), \ldots,\left(j_{b-1}, j_{b-1}\right),\left(j_{b}, j_{b}\right)=(n, n) .
$$

We define the bounce statistic bounce $(\pi)$ to be the sum

$$
\operatorname{bounce}(\pi)=\sum_{i=1}^{b-1} n-j_{i} \text {. }
$$

Also, let $a_{i}=a_{i}(\pi)$ denote the number of squares in the ith row (from the bottom) which are to the left of the path and strictly above the diagonal $x=y$, and set area $(\pi)=\sum_{i=1}^{n} a_{i}$. For the path in Figure 6, we have bounce $=19$, area $=15$, and

$$
\left(a_{1}, a_{2}, \ldots, a_{11}\right)=(0,1,2,1,2,0,1,1,2,3,2) .
$$

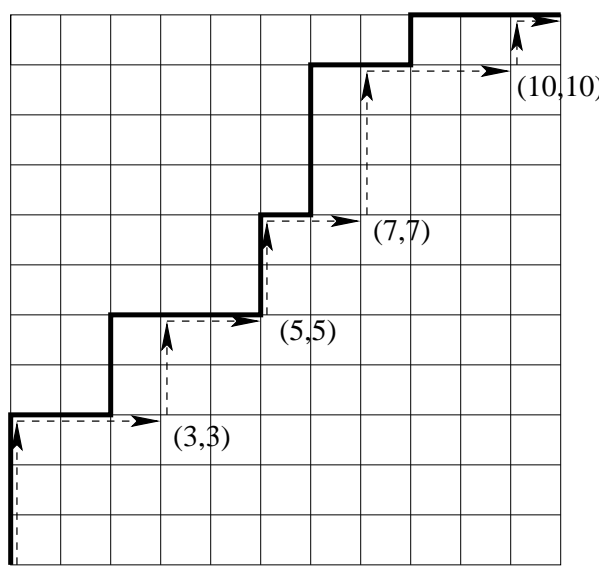

Figure 6: The bounce path (dotted line) of a Dyck path (solid line). The bounce statistic equals $11-3+11-5+11-7+11-10=8+6+4+1=19$, and area $=15$.

Theorem 1 ([GH01], [GH02]).

$$
C_{n}(q, t)=\sum_{\pi \in L_{n, n}^{+}} q^{\text {area( }(\pi)} t^{\text {bounce }(\pi)} .
$$


There is another, equivalent way of describing $C_{n}(q, t)$ which was discovered by M. Haiman [Hai00]. Given a path $\pi$ as above, we say rows $i$ and $j$ form an inversion if

$$
1 \leq i<j \leq n \text { and either } a_{i}=a_{j} \text { or } a_{i}=a_{j}+1 .
$$

Letting $\operatorname{dinv}(\pi)$ denote the number of such inversion pairs, then

$$
C_{n}(q, t)=\sum_{\pi \in L_{(n, n)}^{+}} q^{\operatorname{dinv}(\pi)} t^{\operatorname{area}(\pi)} .
$$

To prove that (22) and (20) are equivalent, we give an abbreviated description of the "zeta map" between Dyck paths, which has the property that

$$
\begin{aligned}
& \operatorname{dinv}(\pi)=\operatorname{area}(\zeta(\pi)) \\
& \operatorname{area}(\pi)=\operatorname{bounce}(\zeta(\pi)) .
\end{aligned}
$$

This map is described in more detail in [Hag08, pp. 50-51].

Say $b-1$ is the length of the longest row of $\pi$, i.e. the maximal value of an $a_{i}$. The lengths of the bounce steps of $\zeta$ will be $\alpha_{1}, \ldots, \alpha_{b}$, where $\alpha_{i}$ is the number of rows of length $i-1$ in $\pi$. To construct the actual path $\zeta$, place a pen at the lattice point with $(x, y)$-coordinates $(0,0)$, and draw a line straight up to $\left(0, \alpha_{1}\right)$. Now look at the subsequence of the $a_{i}$ consisting of only those $a_{i}$ which are either 0 or 1 . Travel through this subsequence, and each time you encounter a 0, draw an $E$ step, and each time you encounter a 1, draw a $N$ step. You should now be at the lattice point $\left(\alpha_{1}, \alpha_{1}+\alpha_{2}\right)$. Next look at the subsequence of the $a_{i}$ consisting of only those $a_{i}$ which are either 1 or 2 . Travel through this subsequence, and each time you encounter a 1 , draw an $E$ step, and each time you encounter a 2, draw a $N$ step. You should now be at the lattice point $\left(\alpha_{1}+\alpha_{2}, \alpha_{1}+\alpha_{2}+\alpha_{3}\right)$. Next, consider the subsequence of 2's and 3's, etc., and continue in this way until you arrive at $(n, n)$. See Figure 7.

It is easy to see this map is a bijection: given $\zeta(\pi)$, from the bounce path we can determine the multiset of row lengths of $\pi$. We can then build up the area sequence of $\pi$; from the portion of the path between $\left(\alpha_{1}, \alpha_{1}+\alpha_{2}\right)$ we can see how to interleave the rows of lengths 0 and 1 , and then we can insert the rows of length 2 into the area sequence, etc.. We leave it as an exercise for the reader to verify (23) holds.

There is a more general form of Theorem 1 due to the author. Define the reading order of the rows of $\pi$ to be the order in which the rows are listed by decreasing value of $a_{i}$, where if two rows have the same $a_{i}$-value, the row above is listed first. For the path on the left in Figure 7, the reading order is

row 6 , row 8 , row 5 , row 4 , row 3 , row 7 , row 2 , row 1 .

Finally let $b_{k}=b_{k}(\pi)$ be the number of inversion pairs as in (21) which involve the $k$ th row in the reading order and rows that occur before it in the reading order, and set $b_{0}=-1$. For the path on the left in Figure 7, we have

$$
\left(b_{0}, b_{1}, \ldots, b_{8}\right)=(-1,0,1,1,2,3,3,1,0) .
$$

Let

$$
C_{n}(q, t, z)=\sum_{d=0}^{n} z^{d}\left\langle\nabla e_{n}, e_{n-d} h_{d}\right\rangle
$$



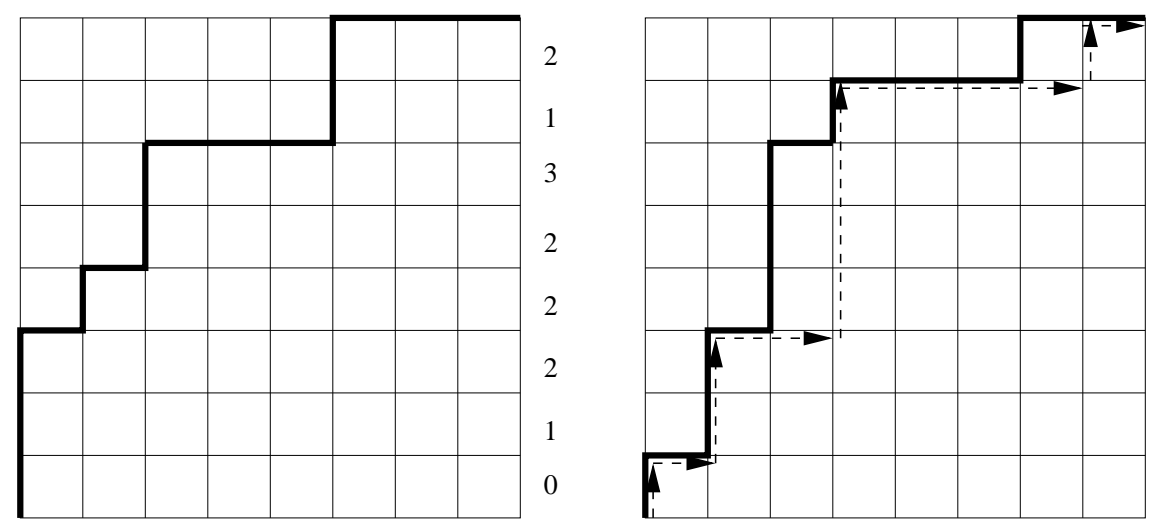

Figure 7: A path $\pi$ with row lengths to the right, and the image $\zeta(\pi)$.

Theorem 2 [Hag04]

$$
C_{n}(q, t, z)=\sum_{\pi \in L_{n, n}^{+}} q^{\operatorname{dinv}(\pi)} t^{\operatorname{area}(\pi)} \prod_{\substack{1 \leq i \leq n \\ b_{i}>b_{i-1}}}\left(1+z / q^{b_{i}}\right) .
$$

Note that in the product on the right hand side of (27), there is always a factor of $1+z$ corresponding to $i=1$. Furthermore, since by construction dinv $=\sum_{i \geq 1} b_{i}$, the coefficient of $z^{d}$ in (27) is clearly in $\mathbb{N}[q, t]$.

We mention that (26) implies

$$
\sum_{d=0}^{n-1} z^{d}\left\langle\nabla e_{n}, s_{d+1,1^{n-d-1}}\right\rangle=C_{n}(q, t, z) /(1+z) .
$$

\section{Corollary 1}

$$
C_{n}(q, t, z)=\sum_{\pi \in L_{(n, n)}^{+}} q^{\operatorname{area}(\pi)} t^{\text {bounce }(\pi)} \prod_{\substack{a_{i}>a_{i-1} \\ 1 \leq i \leq n}}\left(1+z / q^{a_{i}}\right),
$$

where we set $a_{0}=-1$.

Proof. (Sketch). For $1 \leq i \leq n$, Let $a_{i}^{\prime}(\pi)$ be the number of squares in the $i$ th column (from the right) which are below the path $\pi$ and strictly above the diagonal $x=y$, and set $a_{0}^{\prime}=-1$. It is easy to see that

$$
b_{i}(\pi)=a_{i}^{\prime}(\zeta(\pi)) .
$$

Note that terms in (29) where $a_{i}>a_{i-1}$ correspond to consecutive $N$ steps of $\pi$. By an inductive argument you can identify such pairs of $N$ steps with corresponding pairs of $E$ steps, showing that

$$
\prod_{\substack{a_{i}>a_{i-1} \\ 1 \leq i \leq n}}\left(1+z / q^{a_{i}}\right)=\prod_{\substack{a_{i}^{\prime}>a_{i-1}^{\prime} \\ 1 \leq i \leq n}}\left(1+z / q^{a_{i}^{\prime}}\right)
$$


Theorem 2 is often called the $q, t$-Schröder Theorem, since values of $i$ in (27) for which $b_{i}>b_{i-1}$ correspond to the tops of columns in $\pi$. Hence choosing a given term in (27) which contributes to the overall coefficient of $z^{d}$ corresponds to a selection of $d$ column tops. If we replace each of these column tops by a diagonal step, we get a so-called Schröder path, with $q, t$-weights.

The polynomial $C_{n}(q, t,-a)$ has received more attention recently after E. Gorsky noticed that it equals the $m=n+1$ case of the modified superpolynomial $\tilde{P}_{T(m, n)}(a, q, t)$, as mentioned in Section 1. Gorsky and Negut recently obtained a few explicit formulas for $\tilde{P}_{T(m, n)}(a, q, t)$ which we now discuss.

\subsection{An extension of $\nabla e_{n}$}

As mentioned in Section 1, given any relatively prime pair $(m, n)$ of positive integers, there is an associated operator $Q_{(m, n)}$ on symmetric functions which has arisen from the study of the Elliptic Hall algebra and associated objects. We refer the reader to [BGLX14a] and [BGLX14b] for a concrete, recursive description of these operators. By $Q_{(m, n)}(-\mathbf{1})^{\mathbf{n}}$ we mean the action of $Q_{(m, n)}$ on the constant $(-1)^{n}$. Then

$$
Q_{(n+1, n)}(-\mathbf{1})^{\mathbf{n}}=\nabla e_{n},
$$

and the symmetric functions $Q_{(m, n)}(-\mathbf{1})^{\mathbf{n}}$ form a natural generalization of $\nabla e_{n}$. There is a growing family of conjectured interpretations and identities for these functions. For example, in [GORS14] it is conjectured that $Q_{(m, n)}(\mathbf{- 1})^{\mathbf{n}}$ equals the bigraded Frobenius series of the finite-dimensional, irreducible module $L_{m / n}$ of the rational Cherednik algebra with parameter $c=m / n$. For $m=k n \pm 1$ this conjecture follows from results of Gordon and Stafford [GS05], [GS06]. We now turn to what is known or conjectured about the combinatorics of $Q_{(m, n)}(-\mathbf{1})^{\mathbf{n}}$, and relations to the superpolynomial.

\subsection{Tesler Matrices}

There is an explicit formula for $Q_{m, n}(-\mathbf{1})^{\mathbf{n}}$ due to Gorsky and Negut, expressed in terms of Tesler matrices. These matrices have found previous application to the study of diagonal harmonics [Hag11], [AGH $\left.{ }^{+} 12\right]$, [GHX12].

Theorem 3 (Gorsky, Negut [GN13]) For any pair of positive, relatively prime integers ( $m, n)$,

$$
Q_{m, n}(\mathbf{- 1})^{\mathbf{n}}=\sum_{C \in \operatorname{Tes}(m, n)} \prod_{i=1}^{m} e_{c_{i i}} \prod_{\substack{1 \leq i<m \\ c_{i, i+1}>0}}\left(\left[c_{i, i+1}+1\right]_{q, t}-\left[c_{i, i+1}\right]_{q, t}\right) \prod_{\substack{2 \leq i+1<j \leq m \\ c_{i, j}>0}}(-M)\left[c_{i, j}\right]_{q, t} .
$$

Here Tes $(m, n)$ is the set of $m \times m$ upper-triangular matrices $C$ of nonnegative integers satisfying

$$
c_{i, i}+\sum_{j>i} c_{i, j}-\sum_{j<i} c_{j, i}=\left\lfloor\frac{i n}{m}\right\rfloor-\left\lfloor\frac{(i-1) n}{m}\right\rfloor, \quad 1 \leq i \leq m,
$$

$[k]_{q, t}=\left(t^{k}-q^{k}\right) /(t-q)$, and as before $M=(1-q)(1-t)$. 
Example 3 Note that when $m=n+1$ the conditions (31) reduce to all the "hook sums" $\sum_{j \geq i} c_{i j}-\sum_{j<i} c_{j i}$ equal 1 except for the first which equals 0 . Having a first hook sum equal to 0 forces the first row to be all zeros, and so

$$
\operatorname{Tes}(3,2)=\left\{\left[\begin{array}{lll}
0 & 0 & 0 \\
0 & 1 & 0 \\
0 & 0 & 1
\end{array}\right],\left[\begin{array}{lll}
0 & 0 & 0 \\
0 & 0 & 1 \\
0 & 0 & 2
\end{array}\right]\right\} .
$$

For the leftmost matrix above, the corresponding weight from (30) is $e_{1} e_{1}$ while the weight for the rightmost matrix is $e_{2}(q+t-1)$. Adding these together we get

$$
Q_{(3,2)}(-\mathbf{1})^{2}=e_{1} e_{1}+e_{2}(q+t-1)=s_{2}+(q+t) s_{1^{2}}
$$

in agreement with Example 2.

Example 4 The modified superpolynomial $\tilde{P}_{(m, n)}(q, t, a)$ can be defined as

$$
\tilde{P}_{(m, n)}(q, t, a)=\sum_{d=0}^{n}(-a)^{d}\left\langle Q_{(m, n)}(-\mathbf{1})^{\mathbf{n}}, e_{n-d} h_{d}\right\rangle,
$$

i.e. the generating function for the hook shapes in $Q_{(m, n)}(-\mathbf{1})^{\mathbf{n}}$. Now given a symmetric function $f$ expressed as a polynomial in the $e_{k}$, the scalar product $\left\langle f, e_{n-d} h_{d}\right\rangle$ can be found by replacing each $e_{k}$ by $1+z$, and taking the coefficient of $z^{d}$ in the resulting expression. Hence a formula for $\tilde{P}_{(m, n)}(a, q, t)$ can be obtained by replacing each $e_{c_{i, i}}$ in (30) by $1-a$.

In [ORS12] and [GORS14] a conjectured combinatorial expression for the superpolynomial of the $(m, n)$ torus knot is given, which we now describe (in terms of the modified superpolynomial). In the appendix of [ORS12] it is shown that this conjecture reduces to the $q, t$-Schröder theorem when $m=n+1$.

Let $\operatorname{Grid}(m, n)$ be the $n \times m$ grid of labelled squares whose upper-left-hand corner square is labelled with $(n-1)(m-1)-1$, and whose labels decrease by $m$ as you go down columns and by $n$ as you go across rows. For example,

$$
\operatorname{Grid}(3,7)=\begin{array}{|l|c|r|}
\hline 11 & 4 & -3 \\
\hline 8 & 1 & -6 \\
\hline 5 & -2 & -9 \\
\hline 2 & -5 & -12 \\
\hline-1 & -8 & -15 \\
\hline-4 & -11 & -18 \\
\hline-7 & -14 & -21 \\
\hline
\end{array}
$$

To the corners of the squares of $\operatorname{Grid}(m, n)$ we associate Cartesian coordinates, where the lowerleft-hand corner of the grid has coordinates $(0,0)$, and the upper-right-hand-corner of the grid $(m, n)$. One finds the set of paths $L_{(m, n)}^{+}$which never go below the diagonal $m y=n x$ is the same as the set of $\pi$ for which none of the squares with negative labels are above $\pi$. For a given $\pi$, we let $\operatorname{area}(\pi)$ denote the number of squares in $\operatorname{Grid}(m, n)$ with positive labels which are below $\pi$. Furthermore, let $\operatorname{dinv}(\pi)$ denote the number of squares in $\operatorname{Grid}(m, n)$ which are above $\pi$ and whose arm and leg lengths satisfy

$$
\frac{a}{l+1}<\frac{m}{n}<\frac{a+1}{l}
$$


Here by the arm and leg lengths of a square $s$ we mean the distances from $s$ to $\pi$ in the easterly and southerly directions, respectively. For example, if $(m, n)=(3,7)$ and $\pi=N N N N N E E N N E$, then $\operatorname{area}(\pi)=2$ (corresponding to the squares with labels 2 and 5 ). Also, $\operatorname{dinv}(\pi)=2$; the squares with labels $11,8,4,1$ have $a=l=1 ; a=1, l=0 ; a=0, l=1 ; a=l=0$, respectively, and so the squares with labels 8 and 11 do not satisfy (34), while the squares with labels 1 and 4 do.

Given $\pi \in L_{(m, n)}^{+}$, let $R(\pi)$ denote the set of labels of squares which are at the top of some column of $\pi$. Say these labels occur in columns $c_{1}, c_{2}, \ldots, c_{k}$ as we move left to right. Then for $1 \leq i \leq k$, let $t_{i}$ denote the label of the square which is in the same row as the square at the top of column $c_{i}$, and is also in column $c_{i+1}$, and set $T(\pi)=\left\{t_{1}, t_{2}, \ldots, t_{k-1}\right\}$. For example, if $\pi$ is the path on the left of Figure 8, then

$$
\begin{aligned}
& R(\pi)=\{-3,1,5\} \\
& T(\pi)=\{-6,-2\} .
\end{aligned}
$$

Now form a vector $\alpha(\pi)=\left(\alpha_{1}, \ldots, \alpha_{k}\right)$ consisting of the elements of $R(\pi)$ in decreasing order, and let $\gamma_{i}(\pi)$ denote the number of elements of $R(\pi)$ which are larger than $\alpha_{i}$, minus the number of elements of $T(\pi)$ which are larger than $\alpha_{i}$. For the example of $(35)$, we have $\alpha=(5,1,-3)$, and so $\gamma_{1}=0-0=0, \gamma_{2}=1-0=1, \gamma_{3}=2-1=1$. Furthermore set $\gamma_{0}=-1$.

Conjecture 1 [ORS12] For any pair $(m, n)$ of positive, relatively prime integers,

$$
\tilde{P}_{T(m, n)}(a, q, t)=\sum_{\pi \in L_{m, n}^{+}} q^{\operatorname{dinv}(\pi)} t^{\operatorname{area}(\pi)} \prod_{\substack{\gamma_{i}>\gamma_{i-1} \\ 1 \leq i \leq k}}\left(1-a / q^{\gamma_{i}}\right) .
$$

\subsection{An Extension of the Zeta Map}

There is a candidate extension of the zeta map of Figure 7 which can be described as follows. Given a path $\pi \in L_{(m, n)}^{+}$, call the set of corners of grid squares which are touched by $\pi$ the "vertices" of $\pi$. Next define $S(\pi)$ to be the set consisting of the labels of those squares whose upper-left-hand corners are vertices of $\pi$. A given label in $S(\pi)$ is called an $N$ label if the vertex associated to it is the start of an $N$ step, otherwise it is called an $E$ label. For example, if $\pi$ is the path on the left in Figure 8, then

$$
\pi=N N N N N E N E N E \quad S(\pi)=\{-10,-7,-4,-1,2,5,-2,1,-6,-3\} .
$$

We now define the "sweep map" of [ALW14], denoted $\zeta$, from $L_{(m, n)}^{+}$to $L_{(m, n)}^{+}$as follows: order the elements of $S(\pi)$ in increasing order to create a vector of labels $D(\pi)=\left(d_{1}, d_{2}, \ldots, d_{m+n}\right)$. Then create a path $\phi(\pi)$ by defining the $i$ th step of $\phi(\pi)$ to be an $N$ step if $d_{i}$ is an $N$ label, and an $E$ step if $d_{i}$ is an $E$ label. For the example in (38), we have

$$
D(\pi)=(-10,-7,-6,-4,-3,-2,-1,1,2,5) \quad \phi(\pi)=N N N N E N N E N E .
$$

We note that when $m=n+1$, paths in $L_{(m, n)}^{+}$are in bijection with paths in $L_{(n, n)}^{+}$, and that the sweep map reduces to the $\zeta$ map of Figure 7. 


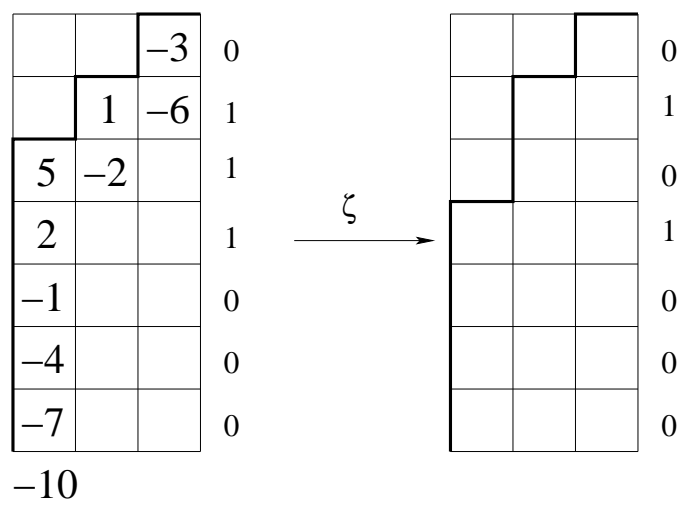

Figure 8: The sweep map.

\section{The Rational Shuffle Conjecture}

There is a nice conjecture for the expansion of the symmetric function $Q_{(m, n)}(-\mathbf{1})^{\mathbf{n}}$ into monomials, which is described in [GN13] and also [BGLX14a]. If $m=n+1$ it reduces to the shuffle conjecture from $\left[\mathrm{HHL}^{+} 05 \mathrm{c}\right]$. Let an $(m, n)$-parking function be a path $\pi \in L_{(m, n)}^{+}$together with a placement of the integers 1 through $n$ (called cars) just to the right of the $\mathrm{N}$ steps of $\pi$, with strict decrease down columns. For such a pair $P$, we let $\operatorname{rank}(j)$ be the label of the square that contains $j$, and we set

$$
\operatorname{tdinv}(P)=\mid\{(i, j): 1 \leq i<j \leq n \text { and } \operatorname{rank}(i)<\operatorname{rank}(j)<\operatorname{rank}(i)+m\} \mid .
$$

Furthermore we let the reading word $\operatorname{read}(P)$ be the permutation obtained by listing the cars by decreasing order of their ranks. For example, for the $(3,7)$-parking function of Figure 9 , tdinv $=3$, with inversion pairs formed by pairs of cars $(6,7),(4,6)$, and $(2,4)$, and the reading word is 7642531 .

\begin{tabular}{|l|l|l|}
\hline & & 5 \\
\hline & 6 & \\
\hline & 2 & \\
\hline 7 & & \\
\hline 4 & & \\
\hline 3 & & \\
\hline 1 & & \\
\hline
\end{tabular}

Figure 9: A $(3,7)$-parking function.

Let $\operatorname{maxtdinv}(\pi)$ be tdinv of the parking function for $\pi$ whose reading word is the reverse of the identity, and for any parking function $P$ for $\pi$ set

$$
\operatorname{dinv}(P)=\operatorname{dinv}(\pi)+\operatorname{tdinv}(P)-\operatorname{maxtdinv}(\pi) .
$$


Then one formulation of the rational shuffle conjecture is the following

Conjecture $\mathbf{2}$ For any pair of relatively prime positive integers $(m, n)$ and any pair of compositions $\alpha, \beta$ with $\sum_{i} \alpha+\sum_{j}$ beta $=n$, we have

$$
\left\langle Q_{(m, n)}(-\mathbf{1})^{\mathbf{n}}, e_{\alpha} h_{\beta}\right\rangle=\sum_{(m, n) \text { parking functions } P} q^{\operatorname{dinv}(P)} t^{\operatorname{area}(\pi)},
$$

where the sum is over all $(m, n)$ parking functions $P$ whose reading word is a shuffle of decreasing sequences of lengths $\alpha_{1}, \alpha_{2}, \ldots$ and increasing sequences of lengths $\beta_{1}, \beta_{2}, \ldots$ Here $e_{\alpha}=\prod_{i} e_{\alpha_{i}}$, and $h_{\beta}=\prod_{i} h_{\beta_{i}}$.

We note that the values of $\left\langle Q_{(m, n)}(-\mathbf{1})^{\mathbf{n}}, h_{\beta}\right\rangle$ for all partitions $\beta$ define $Q_{(m, n)}(-\mathbf{1})^{\mathbf{n}}$ as a symmetric function, so at first glance it seems the conditions in $(42)$ over determine $Q_{(m, n)}(-\mathbf{1})^{\mathbf{n}}$. See [Hag08, p. 99] for an explanation of why the case of $\alpha=\emptyset$ in (42) implies the case of general $\alpha$. We also mention that the motivation for the right-hand-side of (42) comes largely from work of Hikita [Hik14]. He showed that the bigraded Frobenius series of a certain module arising from affine Springer fibers is the same as the bigraded Frobenius series which the rational shuffle conjecture predicts for $Q_{(m, n)}(\mathbf{- 1})^{\mathbf{n}}$. Hence one approach to the rational shuffle conjecture is to try and connect the $Q_{(m, n)}(-\mathbf{1})^{\mathbf{n}}$ to Hikita's affine Springer fiber modules directly.

Example 5 If $\alpha=(n-d)$ and $\beta=(d)$, the left-hand-side of (42) becomes

$$
\left\langle Q_{(m, n)}(-\mathbf{1})^{\mathbf{n}}, e_{n-d} h_{d}\right\rangle .
$$

We claim the right-hand-side of (42) is the coefficient of $w^{d}$ in the right-hand-side of (37). To see why, fix an $(m, n)$ path $\pi$, and let our increasing sequence be $(n-d+1, \ldots, n)$ and our decreasing sequence be $(n-d, \ldots, 2,1)$. Let $P$ be a parking function for $\pi$ whose reading word is a shuffle of these sequences. Note the elements of $(n-d+1, \ldots, n)$ of $P$ must occur at the tops of columns. Call these column tops $c_{1}, \ldots, c_{k}$, and say their rank numbers are $r_{1}, \ldots, r_{k}$. How does dinv $(P)$ differ from dinv $(\pi)$ ? From (41), to answer this question we need to determine $-\operatorname{tdinv}(P)+\operatorname{maxtdinv}(\pi)$. It is not hard to see that this difference is the sum, over all column tops $c_{i}$ for $1 \leq i \leq k$, of the number of cars of $P$ which reside in a column top square whose rank $r$ satisfies $r_{i}<r<r_{i}+m$, and which are also in the increasing sequence. The calculation of the $C_{i}$ from (37) accomplishes this same purpose. Hence the coefficient of $w^{d}$ in the right-hand-side of (37) is the same as that which the rational shuffle conjecture predicts for (43), in agreement with (32).

\section{Open Problems}

Drew Armstrong has coined the phrase "rational Catalan combinatorics" for combinatorial issues connected to paths in $L_{(m, n)}^{+}$and the rational shuffle conjecture [Arm12]. (These slides also introduce the right-hand-side of (42), independently of Hikita's work). In this section we list a few open problems involving rational Catalan combinatorics.

Open Problem 1 Prove that for general coprime $(m, n)$ the sweep map is a bijection from $L_{(m, n)}^{+} \rightarrow L_{(m, n)}^{+}$. 
This problem has been studied by Gorsky, Mazin, and Vazirani [GMV14] and Armstrong, Loehr, and Warrington [ALW14]. See also [AHJ14]. A related question is to find a bounce statistic on paths in $L_{(m, n)}^{+}$which, when combined with area, generates $\left\langle Q_{(m, n)}(\mathbf{- 1})^{\mathbf{n}}, s_{1^{n}}\right\rangle$. In [GMV14] it is shown that the sweep map is a bijection whenever $m=k n+1$ or $m=k n-1$ for some positive integer $k$. We note that in the case $m=k n+1$ Loehr [Loe03], [Loe05] (see also [Hag08][pp. 108-109]) has defined an extension of the bounce statistic, which when combined with area generates the $q, t$-Catalan for $m=k n+1$. For this "paths in a $n \times k n$ rectangle" case there is also an interpretation for the rational shuffle conjecture in terms of a generalization of diagonal harmonics (see $\left[\mathrm{HHL}^{+} 05 \mathrm{c}\right]$ ).

Open Problem 2 Prove that

$$
\sum_{\pi \in L_{(m, n)}^{+}} q^{\operatorname{dinv}(\pi)+(n-1)(m-1) / 2-\operatorname{area}(\pi)}=\frac{1}{[m]}\left[\begin{array}{c}
n+m-1 \\
n
\end{array}\right] .
$$

Here $[m]=\left(1-q^{m}\right) /(1-q)$ and $\left[\begin{array}{c}n+m-1 \\ n\end{array}\right]$ is the q-binomial coefficient.

There is currently no known way to generate the right-hand-side of (44) as a sum over weighted lattice paths. It is known [GN13, p. 28], [GLWX15] that

$$
\left.q^{(n-1)(m-1) / 2}\left\langle Q_{(m, n)}(-\mathbf{1})^{\mathbf{n}}, s_{1^{n}}\right\rangle\right|_{t=1 / q}=\frac{1}{[m]}\left[\begin{array}{c}
n+m-1 \\
n
\end{array}\right],
$$

so the sign character case of the rational shuffle conjecture would imply (44).

Open Problem 3 Refine the rational shuffle conjecture by finding a combinatorial prediction for the coefficient of an arbitrary Schur function in $Q_{(m, n)}(-\mathbf{1})^{\mathbf{n}}$.

We mention that one consequence of the rational shuffle conjecture is that $Q_{(m, n)}(-\mathbf{1})^{\mathbf{n}}$ can be decomposed into a sum of symmetric functions, one for each $(m, n)$ path. These symmetric functions are special cases of LLT polynomials, specifically they are LLT products of vertical strips - see [GM15], and [Hag08, Chapter 6] for an explanation of why this is true for the $m=n+1$ case. Hence understanding the Schur coefficients of $Q_{(m, n)}(-\mathbf{1})^{\mathbf{n}}$ is embedded in the famous problem of understanding the Schur coefficients of LLT polynomials. (It is also known [HHL05b], [HHL05a], [Hag08, Appendix A] that the modified Macdonald polynomials $\tilde{H}_{\mu}(X ; q, t)$ are a sum of LLT products of ribbon shapes. It is still an open question to understand the Schur coefficients in this case combinatorially.

Open Problem 4 The constructions of Aganagic and Shakirov and of Cherednik for torus knot invariants actually depend on a "coloring" by a partition $\lambda$ in addition to the parameters a, $q, t$ and $m, n$. The partition $\lambda$ is identified with an irreducible representation. The case we have considered in this chapter corresponds to the case $\lambda=(1)$, consisting of a single part of size $1 r$., There is no known explicit formula for any other $\lambda$, although there is an algorithm to compute them [GN13]. It would be quite interesting to have a conjectured combinatorial formula for the superpolynomial for more general $\lambda$. 
Open Problem 5 In [BGLX14a], the "compositional rational shuffle conjecture" is introduced, which includes both the rational shuffle conjecture and the compositional shuffle conjecture from [HMZ12] (which involves $(n, n)$-paths which hit the diagonal $x=y$ in specified places) as special cases. This conjecture is based on the construction of a version of the $Q_{(m, n)}$ operators for general non-coprime $(m, n)$. Can this be used to define a superpolynomial invariant for $(m, n)$ torus links? Is there a Tesler matrix expression for this more general case?

\section{References}

$\left[\mathrm{AGH}^{+} 12\right]$ D. Armstrong, A. Garsia, J. Haglund, B. Sagan, and B. Rhoades. Combinatorics of Tesler matrices in the theory of parking functions and diagonal harmonics. Journal of Combinatorics, 3:451-494, 2012.

[AHJ14] Drew Armstrong, Christopher R. H. Hanusa, and Brant C. Jones. Results and conjectures on simultaneous core partitions. European J. Combin., 41:205-220, 2014.

[Ale28] J. W. Alexander. Topological invariants of knots and links. Trans. Amer. Math. Soc., 30(2):275-306, 1928.

[ALW14] D. Armstrong, N. Loehr, and G. Warrington. Rational parking functions and Catalan numbers. Preprint on the math arXiv:1403.1845, 2014.

[Arm12] D. Armstrong. Rational Catalan combinatorics 1. Slides from a talk at the JMM 2012 meetings, 2012.

[AS11] M. Aganagic and S. Shakirov. Knot homology from refined Chern-Simons theory. Preprint on the math arXiv:1105.5117, 2011.

[AS12] Mina Aganagic and Shamil Shakirov. Refined Chern-Simons theory and knot homology. In String-Math 2011, volume 85 of Proc. Sympos. Pure Math., pages 3-31. Amer. Math. Soc., Providence, RI, 2012.

[BGLX14a] F. Bergeron, A. M. Garsia, E. Leven, and G. Xin. Compositional $(k m, k n)$-shuffle conjectures. Preprint on the math arXiv:1404.4616, 2014.

[BGLX14b] F. Bergeron, A. M. Garsia, E. Leven, and G. Xin. Some remakable new plethystic operators in the thoery of Macdonald polynomials. Preprint on the math arXiv:1405.0316, 2014.

[BS12] Igor Burban and Olivier Schiffmann. On the Hall algebra of an elliptic curve, I. Duke Math. J., 161(7):1171-1231, 2012.

[Che13] Ivan Cherednik. Jones polynomials of torus knots via DAHA. Int. Math. Res. Not. IMRN, (23):5366-5425, 2013.

[Con70] J. H. Conway. An enumeration of knots and links, and some of their algebraic properties. In Computational Problems in Abstract Algebra (Proc. Conf., Oxford, 1967), pages 329-358. Pergamon, Oxford, 1970. 
[DGR06] Nathan M. Dunfield, Sergei Gukov, and Jacob Rasmussen. The superpolynomial for knot homologies. Experiment. Math., 15(2):129-159, 2006.

$\left[\mathrm{FYH}^{+} 85\right]$ P. Freyd, D. Yetter, J. Hoste, W. B. R. Lickorish, K. Millett, and A. Ocneanu. A new polynomial invariant of knots and links. Bull. Amer. Math. Soc. (N.S.), 12(2):239-246, 1985.

[GH93] A. M. Garsia and M. Haiman. A graded representation model for Macdonald polynomials. Proc. Nat. Acad. Sci. U.S.A., 90(8):3607-3610, 1993.

[GH96] A. M. Garsia and M. Haiman. A remarkable $q, t$-Catalan sequence and $q$-Lagrange inversion. J. Algebraic Combin., 5(3):191-244, 1996.

[GH01] A. M. Garsia and J. Haglund. A positivity result in the theory of Macdonald polynomials. Proc. Nat. Acad. Sci. U.S.A., 98:4313-4316, 2001.

[GH02] A. M. Garsia and J. Haglund. A proof of the $q, t$-Catalan positivity conjecture. Discrete Math., 256:677-717, 2002.

[GHX12] A. M. Garsia, J. Haglund, and G. Xin. Constant term methods in the theory of tesler matrices and Macdonald polynomial operators. Annals of Combinatorics, 2012. to appear.

[GLWX15] A. Garsia, E. Leven, N. Wallach, and G. Xin. A new plethystic symmetric function operator and the rational compositional shuffle conjecture at $t=1 / q$. Preprint on the math arXiv: http://arxiv.org/pdf/1501.00631, 2015.

[GM15] E. Gorsky and M. Mazin. Rational parking functions and llt polynomials. Preprint on the math arXiv:1503.04181, 2015.

[GMV14] E. Gorsky, M. Mazin, and M. Vazirani. Affine permutations and rational slope parking functions. Preprint on the math arXiv:1403.0303, 2014.

[GN13] E. Gorsky and A. Negut. Refined knot invariants and Hilbert schemes. Preprint on the math arXiv: http://arxiv.org/pdf/1304.3328, 2013.

[Gor12] E. Gorsky. Private communication, 2012.

[GORS14] Eugene Gorsky, Alexei Oblomkov, Jacob Rasmussen, and Vivek Shende. Torus knots and the rational DAHA. Duke Math. J., 163(14):2709-2794, 2014.

[GS05] I. Gordon and J. T. Stafford. Rational Cherednik algebras and Hilbert schemes. Adv. Math., 198(1):222-274, 2005.

[GS06] I. Gordon and J. T. Stafford. Rational Cherednik algebras and Hilbert schemes. II. Representations and sheaves. Duke Math. J., 132(1):73-135, 2006.

[Hag04] J. Haglund. A proof of the $q, t$-Schröder conjecture. Int. Math. Res. Not., 11:525$560,2004$.

[Hag08] James Haglund. The q,t-Catalan numbers and the space of diagonal harmonics, volume 41 of University Lecture Series. American Mathematical Society, Providence, RI, 2008. With an appendix on the combinatorics of Macdonald polynomials. 
[Hag11] J. Haglund. A polynomial expression for the Hilbert series of the quotient ring of diagonal coinvariants. Adv. Math., 227:2092-2106, 2011.

[Hai94] M. Haiman. Conjectures on the quotient ring by diagonal invariants. J. Algebraic Combin., 3:17-76, 1994.

[Hai00] M. Haiman. Private communication, 2000.

[Hai01] M. Haiman. Hilbert schemes, polygraphs, and the Macdonald positivity conjecture. J. Amer. Math. Soc., 14:941-1006, 2001.

[Hai02] M. Haiman. Vanishing theorems and character formulas for the Hilbert scheme of points in the plane. Invent. Math., 149:371-407, 2002.

[HHL05a] J. Haglund, M. Haiman, and N. Loehr. A combinatorial formula for Macdonald polynomials. Jour. Amer. Math. Soc., 18:735-761, 2005.

[HHL05b] J. Haglund, M. Haiman, and N. Loehr. Combinatorial theory of Macdonald polynomials I: Proof of Haglund's formula. Proc. Nat. Acad. Sci. U.S.A., 102(8):2690-2696, 2005 .

$\left[\mathrm{HHL}^{+} 05 \mathrm{c}\right] \quad$ J. Haglund, M. Haiman, N. Loehr, J. B. Remmel, and A. Ulyanov. A combinatorial formula for the character of the diagonal coinvariants. Duke Math. J., 126:195-232, 2005 .

[Hik14] Tatsuyuki Hikita. Affine Springer fibers of type $A$ and combinatorics of diagonal coinvariants. Adv. Math., 263:88-122, 2014.

[HMZ12] J. Haglund, J. Morse, and M. Zabrocki. A compositional shuffle conjecture specifying touch points of the Dyck path. Canad. J. Math., 64:822-844, 2012.

[Jon85] Vaughan F. R. Jones. A polynomial invariant for knots via von Neumann algebras. Bull. Amer. Math. Soc. (N.S.), 12(1):103-111, 1985.

[Loe03] N. Loehr. Multivariate Analogues of Catalan Numbers, Parking Functions, and their Extensions. Ph.D. Thesis, University of California at San Diego, 2003.

[Loe05] Nicholas A. Loehr. Conjectured statistics for the higher $q, t$-Catalan sequences. Electron. J. Combin., 12:Research Paper 9, 54 pp. (electronic), 2005.

[Mac88] I. G. Macdonald. A new class of symmetric polynomials. Actes du $20^{e}$ Séminaire Lotharingien, Publ. Inst. Rech. Math. Av., 372, 1988.

[Mac95] I. G. Macdonald. Symmetric Functions and Hall Polynomials. Oxford Mathematical Monographs, second ed., Oxford Science Publications. The Clarendon Press Oxford University Press, New York, 1995.

[Neg14] Andrei Negut. The shuffle algebra revisited. Int. Math. Res. Not. IMRN, (22):6242$6275,2014$.

[ORS12] Alexei Oblomkov, Jacob Rasmussen, and Vivek Shende. The Hilbert scheme of a plane curve singularity and the HOMFLY homology of its link. Preprint on the math arXiv:1201.2115, 2012. 
[SV11] O. Schiffmann and E. Vasserot. The elliptic Hall algebra, Cherednik Hecke algebras and Macdonald polynomials. Compos. Math., 147(1):188-234, 2011.

[SV13] Olivier Schiffmann and Eric Vasserot. The elliptic Hall algebra and the $K$-theory of the Hilbert scheme of $\mathbb{A}^{2}$. Duke Math. J., 162(2):279-366, 2013.

[Wik] Wikipedia. Knot theory. 